\title{
Modelling and Performance Analysis of Different Access Schemes in Two-tier Wireless Networks
}

\author{
Xiaohu Ge*, Jorge Martínez-Bauset ${ }^{\dagger}$, Vicente Casares-Giner ${ }^{\dagger}$, Bin Yang*, Junliang Ye* and Min Chen ${ }^{\ddagger}$ \\ ${ }^{*}$ Dept. Electronics \& Information Engineering, Huazhong University of Science \& Technology, Wuhan, China \\ ${ }^{\dagger}$ Dept. de Comunicaciones Universitat Politècnica de València, 46022-Valencia, Spain \\ ${ }^{\ddagger}$ School of Computer Science \& Technology, Huazhong University of Science \& Technology, Wuhan, China \\ Email: xhge@mail.hust.edu.cn, \{jmartinez,vcasares\}@upvnet.upv.es, minchen2012@mail.hust.edu.cn
}

\begin{abstract}
We develop a teletraffic model to evaluate the performance of two typical access schemes in two-tier wireless networks. Two of the main performance parameters are the blocking probability and carried traffic. Numerical results show that the femtocell priority access scheme performs better than the macrocell priority access scheme as it achieves lower blocking probability and carries more traffic.
\end{abstract}

Index Terms-Wireless networks; access scheme; Markov chain; blocking probability

\section{INTRODUCTION}

To accommodate the rapid increase of wireless data traffic in indoor environments, two-tier wireless networks have been proposed to support high throughputs and increase the energy efficiency of wireless communications [1], [2]. The incorporation of femtocells to a cellular network is emerging as a potential solution to achieve these two goals [3]. In this scenario, user terminals meet with the dilemma of choosing an adequate access scheme [4], [5]. For example, first issue a setup requests to the femtocell and if blocked try with the macrocell, or vice versa. We believe that the development of teletraffic models to evaluate the performance of the access scheme in two-tier wireless networks is a problem that deserves further investigation.

Different studies on the performance of femtocell networks have appeared in the literature [6]-[9]. Zhang [6] focuses on the blocking probability in femtocell networks, where a small number of split spectrums to support femtocells were recommended to increase the service availability in the macrocell. Kim et al. [7] derived a per-tier outage probability by introducing a simplified mathematical model which closely approximates the femtocell interference distribution. Chandrasekhar and Andrews [8] analyzed the effect of channel uncertainty parameters. The transmit power level was determined to provide the desired signal-to-interference-plus-noise ratio (SINR) for the indoor cell edge femtocell users and the beam weight to maximize the output SINR of macrocell and femtocell users. By evaluating an interesting networkwide spectral efficiency metric, an uplink capacity analysis and interference avoidance strategy was developed for a twotier femtocell network. Hou and Laurenson [9] showed that the cellular and femtocell heterogeneous network architecture is able to provide a high quality of service (QoS) and signifi- cantly reduce power consumption. Ge et al. [10] investigated the impact of interference on access schemes in wireless networks. Song et al. [11] analyzed the performance of a hybrid wireless network with cellular networks and wireless local area networks. However, most of above studies were carried out deploying a specific cell access scheme in femtocell networks and no comparative results were provided.

Sangiamwong et al. [12] investigated three cell access schemes in heterogeneous networks with Long-Term Evolution (LTE)-Advanced downlink: signal-to-interference plus noise power ratio (SINR)-based access scheme, reference signal received power (RSRP)-based access scheme, and reference signal received quality (RSRQ)-based access scheme. Simulation results showed that the performance of the RSRQbased access scheme for downlink cell-central and cell-edge users performs better than the other two access schemes, in terms of maximizing the cell-edge user throughput. Fooladivanda proposed a simple cell access scheme, i.e., Picocell First (PicoF) that performs much better than two existing cell access scheme, i.e., received signal power access scheme and range extension (RE) access scheme [13]. All above studies of cell priority schemes were based on simulation models. We believe that analytical models from the traffic perspective to analyze the performance of cell priority schemes in two-tier wireless networks have nor received sufficient attention in the literature.

In this paper, we develop a comparative performance evaluation study of two cell access schemes for a two-tier wireless network from a traffic perspective. We refer to these two models as: macrocell priority access scheme and femtocell priority access scheme. Performance parameters, such as blocking probability, carried traffic and the traffic congestion perceived by the femtocell base stations are derived. Also, we validate the analytical models by simulation. The results obtained provide guidelines for the selection of the cell access scheme in two-tier wireless networks.

The rest paper is organized as follows. Section II describes the system model of a two-tier wireless network. In Section III and IV, two continuous-time Markov chain (CTMC) models are proposed to evaluate the performance of the two access schemes that we study. In Section V we define expression for the performance parameter of interest: the blocking probability, carried traffic and traffic congestion. The results of a comparative numerical evaluation study are 


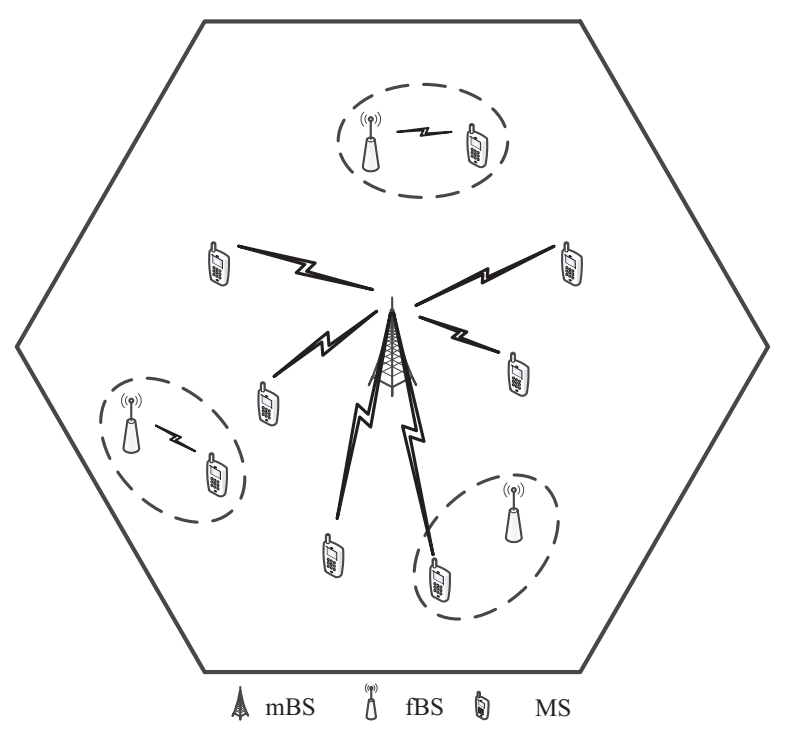

Fig. 1. System model of two-tier wireless network.

shown in Section VI. Finally, Section VII concludes the paper.

\section{SYSTEM MODEL}

A simplified scenario of a two-tier wireless network is shown in Fig. 1, which includes a single macrocell and several femtocells. Three types of functional units are considered in Fig. 1, i.e., the macrocell base station (mBS), femtocell base stations (fBSs) and mobile stations (MSs). The mBS has $C_{m}$ channels and an hexagonal shape coverage area with surface $S_{m}$. There are $N$ femtocells uniformly distributed in the macrocell coverage area, and we assume that their coverage areas do not overlap with each other. We denote by $S_{i}$ the surface of the coverage area of the fBS $i$, and by $C_{i}$ the number of channels associated to it, $1 \leq i \leq N$. For mathematical tractability, we follow the common assumption that calls arrive to the system following a Poisson process of rate $\lambda$. We also assume that call durations are exponentially distributed with rate $\mu$. Accepted calls occupy one channel. Our model does not consider mobility. However it can be easily incorporated at the expense of increasing its complexity. Finally, we assume that a MS is able to access both the mBS and any fBS that falls within its communication range (the location of the MS is within its coverage area).

\section{MODELLING OF MACROCELL PRIORITY ACCESS SCHEME}

We model the macrocell priority access scheme as an overflow system [14]. We consider a system with one macrocell and a single femtocell. Let the mBS channels be the primary group of servers and the fBS channels the secondary group of servers.

A new call arrival is first offered to the mBS. If a free channel is available, the call is carried by the mBS. Otherwise, it is offered to a fBS, provided that the MS is in the coverage area of a fBS. If no free channels are available at the fBS, then

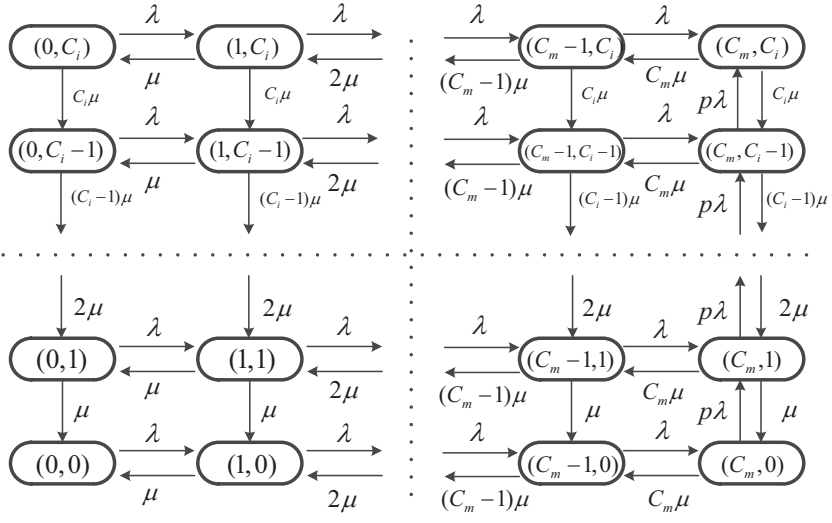

Fig. 2. State transition diagram of macrocell priority access scheme in a system with a one femtocell.

the call is definitely blocked by the two-tier wireless network. We denote by $p=S_{i} / S_{m}$ the probability that a MS is in the coverage area of the fBS $i$.

Let $\left(x_{m}, x_{i}\right)$ be the system state vector, where $x_{m}, 0 \leq$ $x_{m} \leq C_{m}$, is the number of ongoing calls at the primary group (occupied mBS channels), and $x_{i}, 0 \leq x_{i} \leq C_{i}$, the number of ongoing calls at the secondary group (occupied channels at the fBS $i$ ). We note that the system state vector defines an irreducible ergodic CTMC and Fig. 2 illustrates its transition diagram.

The different transitions shown in Fig. 2 are:

1) $\left(x_{m}, x_{i}\right) \rightarrow\left(x_{m}+1, x_{i}\right)$, a new call arrives and it is accepted by the mBS.

2) $\left(x_{m}, x_{i}\right) \rightarrow\left(x_{m}-1, x_{i}\right)$, a mBS call terminates.

3) $\left(C_{m}, x_{i}\right) \rightarrow\left(x_{m}, x_{i}+1\right)$, a new call arrives and it is accepted by the fBS $i$, as all mBS channels are busy.

4) $\left(x_{m}, x_{i}\right) \rightarrow\left(x_{m}, x_{i}-1\right)$, a fBS call terminates.

Let $\pi\left(x_{m}, x_{i}\right)$ be the steady state probability of finding $x_{m}$ ongoing calls at the mBS and $x_{i}$ ongoing calls at the fBS $i$. The stationary distribution is obtained by solving the global balance equations (1).

\section{MODELLING OF FEMTOCELL PRIORITY ACCESS SCHEME}

We model the femtocell priority access scheme as an overflow system. We consider a system with one macrocell and a single femtocell. Let the fBS channels be the primary group of servers and the mBS channels the secondary group of servers.

A new call arrival is first offered to the $\mathrm{fBS} i$, provided that the MS is in the coverage area of this fBS. If a free channel is available at the $\mathrm{fBS} i$, the call is carried by this fBS. If all fBS channels are busy or the MS does not fall within the coverage area of any fBS, then the new call is offered to the $\mathrm{mBS}$. If no free channels are available at the mBS, then the call is definitely blocked by the two-tier wireless network. We denote by $p \lambda$ the arrival rate of calls to the fBS $i$.

As in previous Section, let $\left(x_{m}, x_{i}\right)$ be the system state vector, where $x_{m}, 0 \leq x_{m} \leq C_{m}$, is the number of ongoing 


$$
\left\{\begin{array}{l}
\pi\left(x_{m}, x_{i}\right) \cdot\left(\lambda+x_{m} \mu+x_{i} \mu\right)=\pi\left(x_{m}-1, x_{i}\right) \cdot \lambda+\pi\left(x_{m}+1, x_{i}\right) \cdot\left(x_{m}+1\right) \cdot \mu+\pi\left(x_{m}, x_{i}+1\right) \cdot\left(x_{i}+1\right) \mu \\
\quad \text { if } 0<x_{m}<C_{m}, \quad 0<x_{i}<C_{i}, \\
\pi\left(0, C_{i}\right) \cdot\left(\lambda+C_{i} \mu\right)=\pi\left(1, C_{i}\right) \cdot \mu \\
\pi(0,0) \cdot \lambda=\pi(0,1) \cdot \mu+\pi(1,0) \cdot \mu \\
\pi\left(C_{m}, C_{i}\right) \cdot\left(C_{m} \mu+C_{i} \mu\right)=\pi\left(C_{m}, C_{i}-1\right) \cdot p \lambda+\pi\left(C_{m}-1, C_{i}\right) \cdot \lambda, \\
\pi\left(C_{m}, 0\right) \cdot\left(p \lambda+C_{m} \mu\right)=\pi\left(C_{m}, 1\right) \cdot \mu+\pi\left(C_{m}-1,0\right) \cdot \lambda, \\
\pi\left(x_{m}, C_{i}\right) \cdot\left(\lambda+x_{m} \mu+C_{i} \mu\right)=\pi\left(x_{m}-1, C_{i}\right) \cdot \lambda+\pi\left(x_{m}+1, C_{i}\right) \cdot\left(x_{m}+1\right) \mu, \quad \text { if } 0<x_{m}<C_{m}, \\
\pi\left(x_{m}, 0\right) \cdot\left(\lambda+x_{m} \mu\right)=\pi\left(x_{m}, 1\right) \cdot \mu+\pi\left(x_{m}-1,0\right) \cdot \lambda+\pi\left(x_{m}+1,0\right) \cdot\left(x_{m}+1\right) \mu, \quad \text { if } 0<x_{m}<C_{m}, \\
\pi\left(0, x_{i}\right) \cdot\left(\lambda+x_{i} \mu\right)=\pi\left(1, x_{i}\right) \cdot x_{m} \mu+\pi\left(0, x_{i}+1\right) \cdot\left(x_{i}+1\right) \mu, \quad \text { if } 0<x_{i}<C_{i} \\
\pi\left(C_{m}, x_{i}\right) \cdot\left(C_{m} \mu+x_{i} \mu+p \lambda\right)=\pi\left(C_{m}, x_{i}+1\right) \cdot\left(x_{i}+1\right) \mu+\pi\left(C_{m}-1, x_{i}\right) \cdot \lambda+\pi\left(C_{m}, x_{i}-1\right) \cdot p \lambda \\
\quad \text { if } 0<x_{i}<C_{i} .
\end{array}\right.
$$
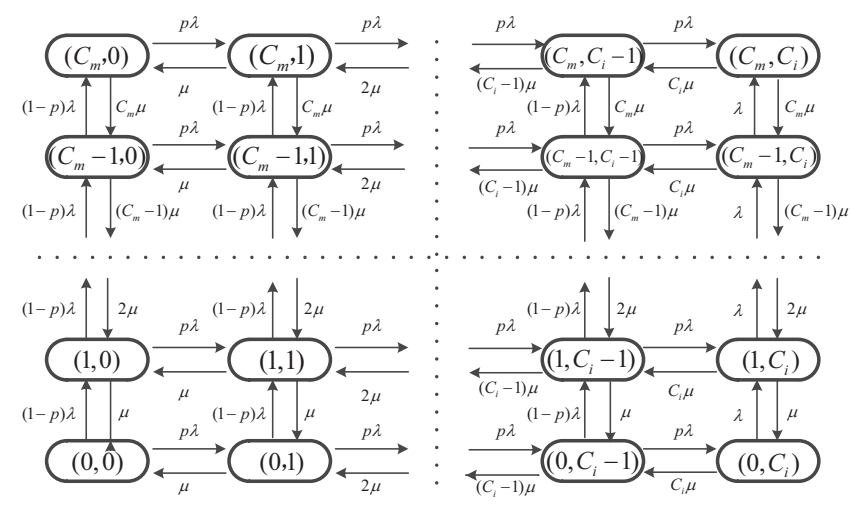

Fig. 3. State transition diagram of femtocell priority access scheme in a system with a one femtocell.

calls at the secondary group (occupied mBS channels), and $x_{i}$, $0 \leq x_{i} \leq C_{i}$, the number of ongoing calls at the primary group (occupied channels of the fBS $i$ ). We note that the system state vector defines an irreducible ergodic CTMC and Fig. 3 illustrates its transition diagram.

The different transitions shown in Fig. 3 are:

1) $\left(x_{m}, x_{i}\right) \rightarrow\left(x_{m}, x_{i}+1\right)$, a new call arrives and it is accepted by the fBS $i$.

2) $\left(x_{m}, x_{i}\right) \rightarrow\left(x_{m}, x_{i}-1\right)$, a fBS call terminates.

3) $\left(x_{m}, x_{i}\right) \rightarrow\left(x_{m}+1, x_{i}\right)$, a new call arrives and it is accepted by the mBS, as all fBS channels are busy or the MS does not fall within the coverage area of the fBS $i$.

4) $\left(x_{m}, x_{i}\right) \rightarrow\left(x_{m}-1, x_{i}\right)$, a mBS call terminates.

Note in Fig. 3 that when there are free channels at fBS $i$ the mBS call arrival rate is $(1-p) \lambda$. However, when all channels at $\mathrm{fBS} i$ are occupied, the mBS call arrival rate is $\lambda$.

Let $\pi\left(x_{m}, x_{i}\right)$ be the steady state probability of finding $x_{m}$ ongoing calls at the mBS and $x_{i}$ ongoing calls at the fBS $i$. The stationary distribution is obtained by solving the global balance equations (2).

\section{PERFORMANCE PARAMETERS}

Considering a system with one mBS macrocell and $N$ fBSs, we denote by $\pi\left(x_{m}, x_{1}, \ldots, x_{N}\right)$ the joint stationary probability of finding $x_{m}\left(x_{i}\right)$ ongoing calls in the mBS (fBS $i)$. For simplicity, we assume that all fBS have the same number of channels and the coverage areas of all femtocells have the same surface, i.e., $C_{i}=C_{f}, S_{i}=S_{f}, \forall i, p=S_{f} / S_{m}$ and $N p \leq 1$. Then, the blocking probability is given by

$$
\begin{aligned}
B P & =(1-N p) \pi\left(C_{m}, *, \ldots, *\right)+p \pi\left(C_{m}, C_{f}, *, \ldots, *\right) \\
& +p \pi\left(C_{m}, *, C_{f}, \ldots, *\right)+\ldots+p \pi\left(C_{m}, *, \ldots, C_{f}\right) \\
& =(1-N p) \pi\left(C_{m}, *, \ldots, *\right)+N p \pi\left(C_{m}, C_{f}, *, \ldots, *\right) .
\end{aligned}
$$

Clearly, a new call is blocked when the corresponding MS is not covered by any of the $N$ fBSs and the mBS has all channels occupied, or when it is covered by a fBS but all channels of the fBS and $\mathrm{mBS}$ are occupied. Note that for symmetry and regardless of the access scheme adopted, the fraction of time with all mBS channels busy and all fBS $i$ channels busy is equal to the fraction of time with all mBS channels busy and all fBS $j$ channels busy, $1 \leq i, j \leq N$, i.e.,

$$
\pi\left(C_{m}, *, \ldots, C_{i}, \ldots, *\right)=\pi\left(C_{m}, *, \ldots, C_{j}, \ldots, *\right) .
$$

For the macrocell priority access scheme, the terms in (3) required to determine the $B P$ can be easily obtain from the two dimensional system defined in Section III. As an example, the stationary probabilities in (3) for a system with $N=2$ can be determined as follows.

$$
\begin{gathered}
\pi\left(C_{m}, *, *\right)=\sum_{i=0}^{C_{f}} \sum_{j=0}^{C_{f}} \pi\left(C_{m}, i, j\right)=\pi_{m}\left(C_{m}\right), \\
\pi\left(C_{m}, C_{f}, *\right)=\sum_{i=0}^{C_{f}} \pi\left(C_{m}, C_{f}, i\right)=\pi_{1}\left(C_{m}, C_{f}\right), \\
\pi\left(C_{m}, *, C_{f}\right)=\sum_{i=0}^{C_{f}} \pi\left(C_{m}, i, C_{f}\right)=\pi_{2}\left(C_{m}, C_{f}\right) .
\end{gathered}
$$




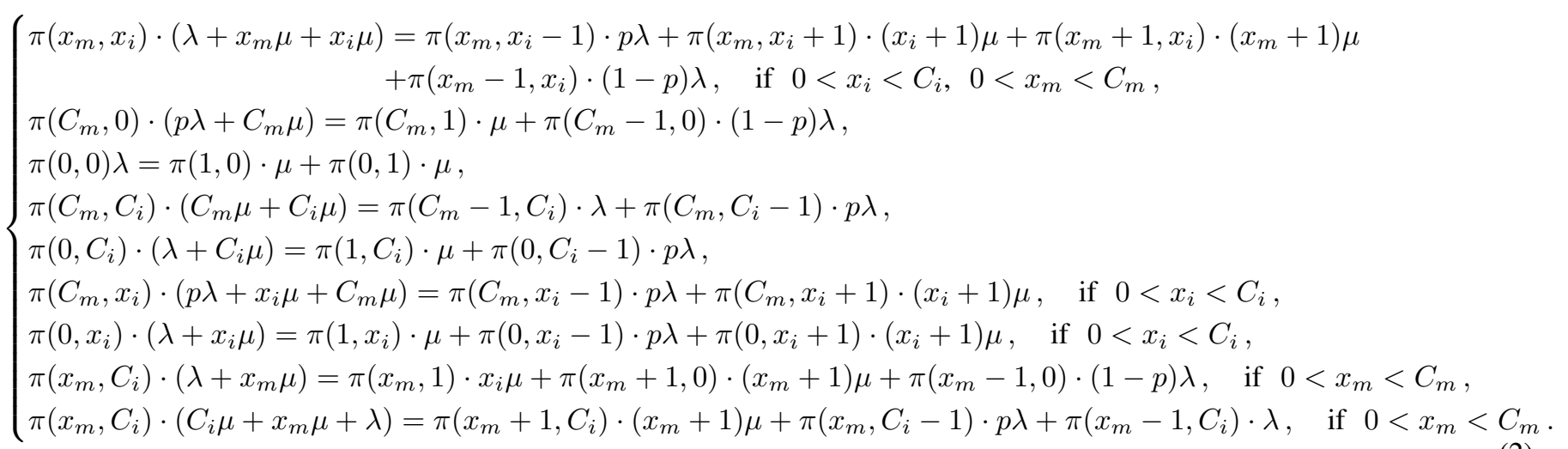

Note that $\pi_{m}\left(C_{m}\right)$ is the blocking probability in a Erlang-B system with $C_{m}$ channels. Also, the probability $\pi_{1}\left(C_{m}, C_{f}\right)$ was already obtained in Section III.

For the macrocell priority access scheme, the total traffic carried by the two-tier system with one mBS and $N$ fBSs is given by [15]

$$
\begin{aligned}
A_{C} & =\sum_{x_{m}=0}^{C_{m}} x_{m} \pi_{m}\left(x_{m}\right)+N \sum_{x_{m}=0}^{C_{m}} \sum_{x_{1}=0}^{C_{f}} x_{1} \pi_{1}\left(x_{m}, x_{1}\right) \\
& =A_{C m}+N A_{C f}
\end{aligned}
$$

where $A_{C m}$ is the traffic carried by the macrocell, $A_{C f}$ is the traffic carried by any femtocell, and the distribution $\pi_{1}\left(x_{m}, x_{1}\right)$ can be obtained with the model of Section III.

Finally, the traffic lost by the macrocell $A_{L m}$ and any femtocell $A_{L m}$, and the traffic congestion perceived by any femtocell $T C_{f}^{M-F}$ are given by

$$
\begin{aligned}
A_{L m} & =A_{O} \pi\left(C_{m}\right), \quad A_{O}=\lambda / \mu, \\
A_{L f} & =A_{O f}-A_{C f}=p A_{L m}-A_{C f} \\
& =p A_{O} \pi\left(C_{m}\right)-\sum_{x_{m}=0}^{C_{m}} \sum_{x_{1}=0}^{C_{f}} x_{1} \pi_{1}\left(x_{m}, x_{1}\right), \\
T C_{f}^{M-F} & =\frac{A_{L f}}{A_{O f}},
\end{aligned}
$$

respectively, where $A_{O}$ is the total traffic offered to the twotier network, $A_{O f}$ is the traffic offered to any femtocell.

For the femtocell priority scheme, we assume that the probabilities $\pi\left(C_{m}, *, \ldots, C_{i}, \ldots, *\right)$ required to determine the blocking probability (3) can be approximated as in (4), (5) and (6), but now $\pi_{i}\left(x_{m}, x_{i}\right)$ are obtained by the model defined in Section IV.

Then, the performance parameters of interest are given by the same expressions provided for the macrocell priority scheme except the traffic congestion, which is given by

$$
T C_{f}^{F-M}=\frac{p A_{O}-\sum_{x_{m}=0}^{C_{m}} \sum_{x_{1}=0}^{C_{f}} x_{1} \pi_{1}\left(x_{m}, x_{1}\right)}{p A_{O}} .
$$

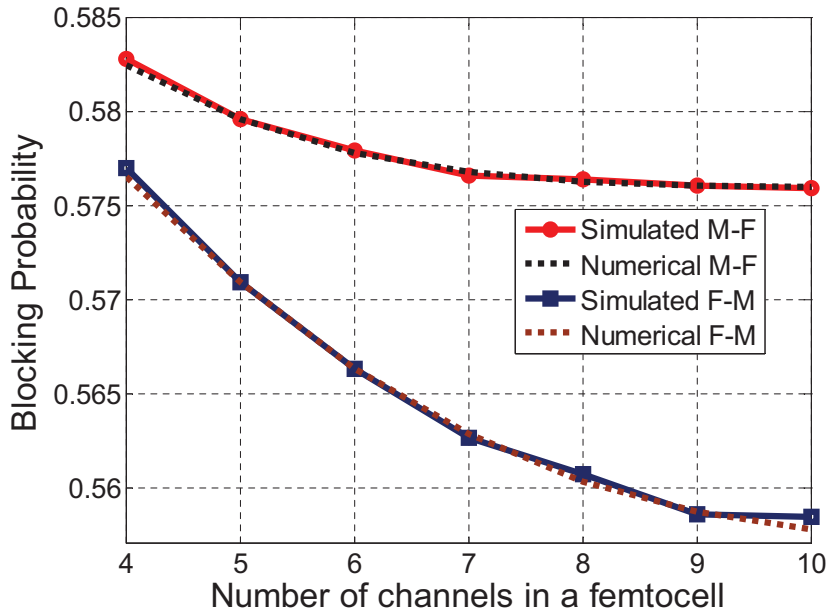

Fig. 4. Blocking probability in a system with one femtocell.

\section{NUMERICAL EVALUATION}

In this Section we compare the performance of the two access schemes studied in two-tier wireless networks. To this end, we define a network with the following configuration: $C_{m}=40, C_{i}=4, \lambda=5, \mu=0.05, S_{m} / S_{i}=20$, and $1 \leq N \leq 20$. The results of the analytical models are validated by simulation.

Figure 4 shows the evolution of the blocking probability with the number of channels of the fBS in a simplified scenario with one mBS and a single fBS. As expected, the blocking probability decreases with the number of channels of the fBS. An interesting observation is that the decrease is more acute for the femtocell priority scheme (F-M) than for the macrocell priority scheme (M-F). Note also that analytical and simulation results match quite well.

Figure 5 shows the evolution of the blocking probability with an increasing number of femtocells. Also as expected, the blocking probability decreases as the number of femtocells increases. An interesting observation is that for a system with only one femtocell the blocking probabilities of both access schemes are very close. However, as the number of femtocells increase, the blocking probability of the F-M scheme decreases 


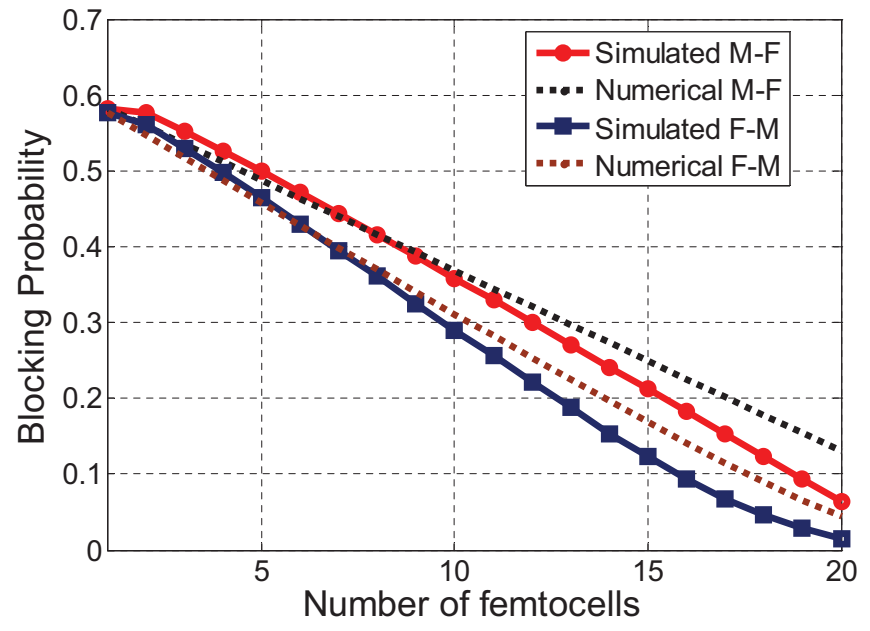

Fig. 5. Blocking probability in a system with multiple femtocells.

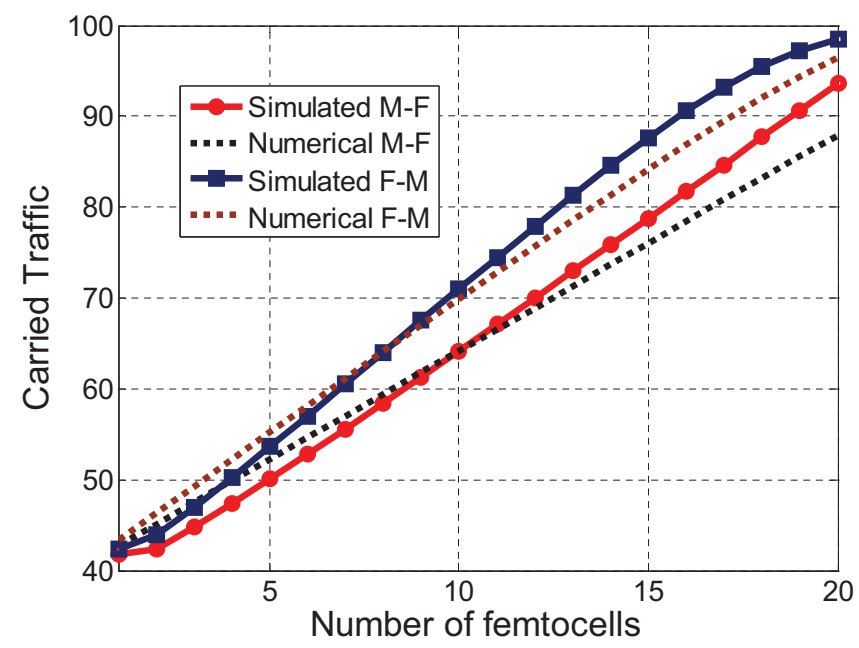

Fig. 6. Total carried traffic in a system with multiple femtocells.

more rapidly than the blocking probability of the M-F scheme.

Figure 6 shows the evolution of the total traffic carried by the two-tier system with an increasing number of femtocells. Again as expected, the total carried traffic increases as the number of femtocells increases. An interesting observation is that the traffic carried by the system with the F-M scheme increases more rapidly than the traffic carried by the system with the M-F scheme with the number of fBSs. Clearly, this trend is in sinthony with the one shown in Fig. 5. Also, note that analytical results closely follow the same trend than the simulation results.

Figure 7 shows the evolution of the traffic congestion rate perceived by a fBS with the number of channels of the fBS, in a simplified scenario with one mBS and a single fBS. Clearly, the fraction of traffic that is lost decreases as the number of channels of the fBS increases. Note that, for a given number of channels of the fBS, the traffic congestion is lower for the F-M access scheme than for the M-F scheme. Finally, note that the traffic congestion perceived by a fBS for when deploying

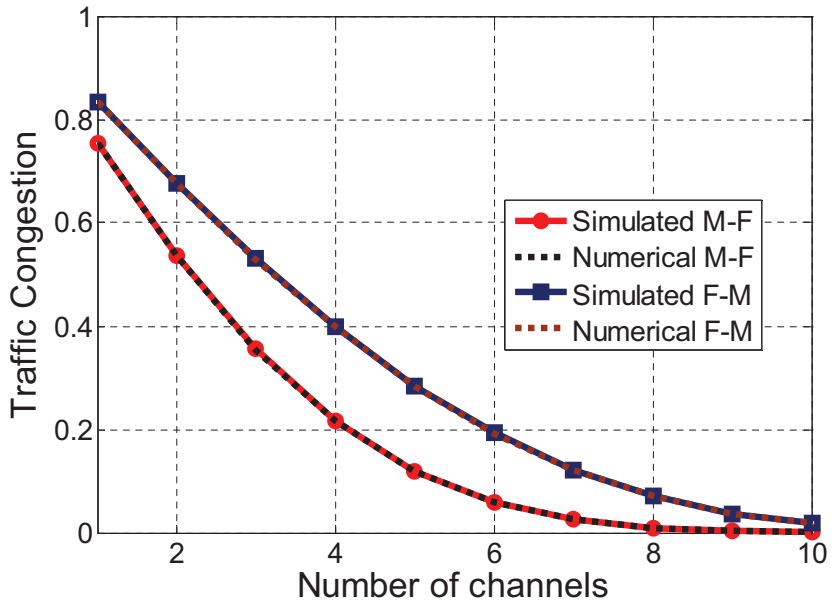

Fig. 7. Traffic congestion perceived by a fBS in a system with a single femtocell.

each of the two access schemes converge to very close values when the number of channels of the fBS is 10 or larger.

Based on above numerical results, we can conclude that the femtocell priority access scheme outperforms the macrocell priority access scheme in terms of the system blocking probability achieved, the total system carried traffic and the traffic congestion.

\section{CONCLUSIONS}

In this paper, we have modeled two cell access schemes in a two-tier wireless network. One is the macrocell priority scheme, where a new call arrival is first offered to the macrocell base station. If blocked by it and the MS is the coverage area of a fBS, then the call is offered to this fBS. The other is the femtocell priority scheme, where a new call arrival is first offered to a fBS if the MS is in the coverage area of the fBS. If blocked by the fBS or the MS is not covered by any $\mathrm{fBS}$, then the new call is offered to the mBS.

We modeled both schemes by two CTMCs and defined three performance parameter: the blocking probability of new calls offered to the system, the total carried traffic by the two-tier system and the traffic congestion perceived by a fBS. The numerical results showed that the femtocell priority access scheme outperforms the macrocell priority access scheme for the performance parameters of interest. Our results provide guidelines for the design of two-tier wireless networks.

\section{ACKNOWLEDGMENTS}

X. Ge, B. Yang and J. Ye would like to acknowledge the support from the National Natural Science Foundation of China (NSFC) (Grant No.: 61210002 and 61271224), National 863 High Technology Program of China (Grant No.: 2009AA01Z239) and the Ministry of Science and Technology (MOST), China, International Science and Technology Collaboration Program (Grant No.:0903), Hubei Provincial Science and Technology Department (Grant No.: 2011BFA004), RCUK for the UK -China Science Bridges Project: R\&D on 
(B) 4G Wireless Mobile Communications, the Korea China Science and Technology Joint Research Center by the National Research Foundation (NRF) under Grant No. 2011-0019905 of the Ministry of Education, Science and Technology (MEST), the Korean government.. X. Ge, J. Martinez-Bauset and V. Casares-Giner acknowledge the support from the EU FP7PEOPLE-IRSES program, project acronym S2EuNet (Grant no.: 247083). V. Casares-Giner contribution was supported by the Spanish Government through project TIN2010-21378C02-02.

\section{REFERENCES}

[1] I. Humar, X. Ge, L. Xiang, et. al., "Rethinking Energy-Efficiency Models of Cellular Networks with Embodied Energy," IEEE Network Magazine, vol. 25, no.3, pp.40-49, Mar. 2011.

[2] C. Wang, T.Q.S. Quek, M. Kountouris, "Throughput optimization, spectrum allocation, and access control in two-tier femtocell networks," IEEE J. Select. Areas Commun., vol. 30, no. 3, pp. 561-574, Apr. 2012.

[3] Z. Lu, Y. Sun, X. Wen, T. Su, D. Ling, "An energy-efficient power control algorithm in femtocell networks," in Proc. International Conference on Computer Science \& Education 2012, pp. 395-400, July 2012.

[4] M. Chen, S. Gonzalez, A. Vasilakos, H. Cao and V. C. M. Leung, "Body Area Networks: A Survey," ACM/Springer Mobile Networks and Applications, Vol. 16, No. 2, pp. 171-193, April 2011.

[5] M. Chen, "MM-QoS for BAN: Multi-Level MAC-Layer QoS Design in Body Area Networks ", IEEE Globecom 2013, Atlanta, Georgia, USA, Dec. 9-13, 2013
[6] Y. Zhang, "Resource sharing of completely closed access in femtocell networks," in Proc. IEEE WCNC 2010, pp. 1-5, Apr. 2010.

[7] Y. Kim, S. Lee and D. Hong, "Performance analysis of two-tier femtocell networks with outage constraints," IEEE Trans. Wireless Commun., vol. 9, no. 9, pp. 2695-2700, Sept. 2010.

[8] V. Chandrasekhar, J. G. Andrews, "Uplink capacity and interference avoidance for two-tier femtocell networks," IEEE Trans. Wireless Commun., vol. 8, no. 7, pp. 3498-3509, July 2009.

[9] Y. Hou and D. I. Laurenson, "Energy efficiency of high QoS heterogeneous wireless communication network," in Proc. IEEE VTC 2010-Fall, pp. 1-5, Sept. 2010

[10] X. Ge, K. Huang, C.-X. Wang, et. al., "Capacity analysis of a multi-cell multi-antenna cooperative cellular network with co-channel interference," IEEE Trans. Wireless Commun., vol. 10, no. 10, pp. 3298-3309, Oct. 2011.

[11] W. Song, H. Jiang and W. Zhuang, "Performance analysis of the WLANfirst scheme in cellular/WLAN interworking," IEEE Trans. Wireless Commun., vol. 6, no. 5, pp. 1932-1952, May 2007.

[12] J. Sangiamwong, Y. Saito, N. Miki, et. al., "Investigation on cell selection methods associated with inter-cell interference coordination in heterogeneous networks for LTE-advanced downlink," in Proc. European Wireless 2011, pp. 1-6, Apr. 2011.

[13] D. Fooladivanda, C. Rosenberg, "Joint resource allocation and user association for heterogeneous wireless cellular networks," IEEE Trans. Wireless Commun., vol. 12, no. 1, pp. 248-257, Jan. 2013.

[14] H. Akimaru, A. Kagechika and H. Takahashi, "Mean and variance of overflow traffic for time dependent inputs," IEEE Trans. Commun., vol. 34, no. 3, pp. 238-243, Mar. 1986.

[15] R.B. Cooper, Queueing Theory, New York: North-Holland, 1981. 